\title{
The emerging role of patient-centered outcomes research in shaping the future of healthcare delivery in India: A brief review
}

\section{Amit Dang, B. N. Vallish ${ }^{1}$}

Founder and CEO MarksMan Healthcare Solutions, HEOR and RWE Consulting, Navi Mumbai,

Maharashtra, ${ }^{1}$ Department of Pharmacology, Velammal Medical College Hospital and Research Institute, Madurai, Tamil Nadu,

India

\section{Address for the Correspondence:} Dr. Amit Dang,

1 - 2, Alfa Garden, Plot No - 26, Sector - 14, Kopar Khairane, Navi Mumbai - 400709 , Maharashtra, India

E-mail: amit.d@ marksmanhealthcare.com

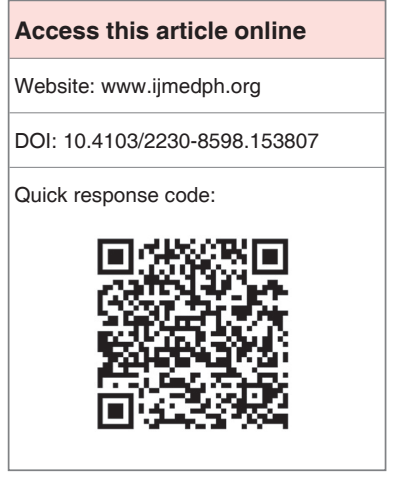

With the Indian patient becoming more empowered with regards to his disease and its treatment options, many Indian physicians still depend only on disease-related outcome measures to take health-care related decisions, and give minimal importance to patient-centered outcomes pertaining to the effect of the healthcare interventions on the patient's well-being. Thus, objective lab values and physician intuition are given more importance than the subjective feeling of well-being that the patient is experiencing following the introduction of the intervention. This review presents a concept of integrating patient-centered and patient-reported data with the existing disease-centered and doctor-reported data for making healthcare-related decisions. This concept, termed patient-centered outcomes research, has already become popular in the Western World.

Key words: Comparative effectiveness research, digital patient, patient-centered outcomes, patient empowerment, patient-reported outcomes

\section{INTRODUCTION}

With the increase in accessibility to the internet, today's patients are more knowledgeable and empowered than those in the past. Patients approaching a healthcare provider for treatment of their medical complaints are increasingly being seen as clients rather than patients. In this background, the treating physician can be seen as a learned vendor who arrives at a diagnosis in his client having a definite set of symptoms and offers him various products to ameliorate the health condition.

However, when it comes to the choice of the best treatment modality, many physicians in India do not take into account the patient preferences. Historically, healthcare decisions and quality assessment is done using objectified measures of the effects of a particular intervention — such as targeted laboratory values or preventable hospitalizations — rather than patient-oriented, subjective measures ${ }^{[1]}$ In reality, the patient is more interested in how better he is feeling after the introduction of the intervention. ${ }^{[2]}$

Thus, during the decision-making process in the Indian healthcare delivery chain, what difference the patient perceives is often given minimal importance, and more weightage is given to what his lab values say, or what the treating clinician feels. This is in stark contrast to other commodities (such as automobiles, consumables, clothing, etc.,) where the client's preferences are given the top priority.

When this scenario is viewed with the background that it is the patient who is paying for the healthcare services, the lacuna becomes much more obvious. The patient is paying for his treatment, but what treatment he gets is defined more by what the treating physician feels (based on lab values or physician's experience and intuition) rather than how the patient feels.

In fact, it has been felt that an assessment of morbidity of any condition can be complete only when both subjective and objective measurement of diseases and disease burden, in addition to an assessment of emotional symptoms in the patient are taken into account. Such multi-dimensional morbidity measurement is particularly essential for assessing the quality of healthcare delivery to patients, especially in complex patient populations. ${ }^{[1]}$ 
There is no doubt that lab values and physician experience and intuition are definitely required while taking healthcare decisions. However, patient preferences and perceptions also should be taken into consideration. This is especially true in two categories of patients:

a. Those who suffer from conditions where there is a lack of consensus amongst the physicians regarding the most optimum treatment option to a particular condition;

b. Patients with multiple, complex and multi-disciplinary healthcare needs. ${ }^{[1]}$

\section{PATIENT-CENTERED OUTCOME RESEARCH}

The current model for defining effectiveness for a particular treatment modality is by analyzing predominantly "disease-centered" data that is almost always "doctor-reported." Optimum and more appropriate model is when the "patient-centered" data are analyzed in addition, and such subjective data should be ideally "patientreported." Such an analysis is the core of the term patient-centered outcome research (PCOR).

Patient-centered outcome research works on the basic premise that, while both clinicians and patients are interested in the best possible solution to the patient's health problems, their priorities differ. A clinician needs objective proof of the effectiveness in therapy, whereas a patient needs subjective relief from the disease symptoms. While a balanced approach is the best suited, we find that an increasing emphasis is given to the objective proof of therapeutic effect. PCOR intends to fill this gap.

\section{THE IMPORTANCE OF PATIENT- CENTERED OUTCOME RESEARCH}

It is increasingly realized that the one-way view of interventions from the physician's viewpoint is incomplete, and it should be complemented with the patient's viewpoint as well, especially because it is the patient who is the final beneficiary of all the interventions, and his voice has to be given its due respect. Further, with the increase in the availability of numerous interventions with similar clinical efficacy for a given condition, favorable profiling of these interventions vis-à-vis the pharmacoeconomic and quality of life (QOL) aspects are increasingly becoming the factors, which identify the favorable intervention.

A well-performed PCOR takes into account the whole wide-eyed viewpoint of an intervention from the patient's side. It aims to collect information with regards to the impact of the intervention on the patient's lifestyle, the economic burden of the intervention, and the patient's perspective of what improvement he is feeling after the initiation of the intervention.

A well-performed PCOR analysis enables the clinician and policy makers to take informed decisions. It also results in better patient adherence and is a vital step in achieving patient empowerment.
Patient-centered outcome research stresses the importance of research informed by the perspectives, interests and values of patients throughout the research process. It particularly emphasizes the viewing of an intervention from the patient perspective and stresses on the measurement of patient-reported outcomes (PROs).

\section{PATIENT-REPORTED OUTCOMES}

The US Food and Drug Administration (USFDA) defines PRO as any report of the status of a patient's health condition that comes directly from the patient, without interpretation of the patient's response by a clinician or anyone else. ${ }^{[3]}$ PROs include a wide variety of variables such as satisfaction scores, symptom relief, well-being, discomfort, productivity assessment, intervention-induced problems, etc. By the virtue of this broader coverage, PROs are starting to replace QOL measures in various places. ${ }^{[4]}$

Patient-reported outcomes allow the patient's voice to emerge within the context of a clinical trial, thereby supplementing clinical indices with patient experiences. PRO data are especially important in chronic, disabling conditions where improvement in QOL forms the most important aspect of therapy. In fact, PROs are among the primary endpoints in clinical trials that evaluate pharmaceutical products of conditions such as irritable bowel syndrome, migraine, pain, insomnia, asthma, psychiatric disorders, and oncology. ${ }^{[5]}$

\section{IMPORTANCE OF PATIENT-REPORTED OUTCOMES}

There has been an increasing call by the authorities that clinicians use patient-relevant endpoints, which are reported by the patients themselves (such as subjective assessments to demonstrate clinical efficacy like pain, depression, asthma symptoms, etc.,) in healthcare decision making.

In addition to physical functions and symptoms, the recording of PROs enables the measurement of outcomes such as global judgments of health, psychological and social well-being, cognitive functioning, role activities, personal constructs, satisfaction with care, health-related quality of life (HRQOL), adherence to medical regimens and clinical trial outcomes. In fact, PRO data may enable early detection of side effects, leading to an improvement in HRQOL and improvement of disease status. ${ }^{[3]}$

Patient-reported outcomes data may support the acceptance of a treatment as an agent offering economic and humanistic value to patients. ${ }^{[3]}$ PROs are also expected to allow the payers of healthcare expenditure (such as government or insurance agencies) to link reimbursement to the effectiveness of treatment. ${ }^{[6]}$

To summarize, a properly collected PRO data has the potential to influence treatment-related decisions made by physicians, patients, hospital formularies, national health authorities, reimbursement agencies, and policy makers. 


\section{REGULATORY ROLE OF PATIENT-REPORTED OUTCOMES}

The use of PROs in clinical trials can provide information regarding patient satisfaction, compliance, and caregiver burden. ${ }^{[7]}$ Realizing the value of the subjective information that can originate from the inclusion of PROs in clinical trials, many industries have started to include PROs routinely in their clinical trials to validate the claims of their pharmaceutical products. In fact, a study in 2004 found out that PROs were included as efficacy endpoints in approximately $30 \%$ of all labels that were reviewed by the USFDA between 1997 and 2002. ${ }^{[8]}$

Following this, the USFDA issued guidance documents for industry in 2006 (with an update in 2009), which are supposed to be followed by industries while developing PRO instruments for supporting label claims. ${ }^{[9]}$ These documents are intended to promote the appropriate development, validation, and use of PRO measures to facilitate favorable regulatory review of label claims in the US. According to the guidance, a claim is defined as "a statement of treatment benefit", and can appear in any section of a medical product's labeling or in advertising and promotional materials. Following the introduction of these documents, it was documented that PRO claims were granted for approximately $24 \%$ of all labels reviewed between January 2006 and December 2010. ${ }^{[5]}$

The present status of PROs in regulatory submission is supplementary to the clinical data and is utilized primarily for validating label claims made by the manufacturer. As the importance of PROs continues to be realized, it may well be possible that PROs are made mandatory for all submissions of drug approval.

\section{PATIENT-CENTERED OUTCOME RESEARCH: PRESENT STATUS}

Patient-centered outcome research is still in infancy in India, and, in fact, only slight progress is made elsewhere in the world. Despite the increased use of digital media for collecting and storing patient information in the Western world, these electronic medical records, disease registries, and administrative data have only rarely collected, or been linked to, PROs. Further, most of those studies that have collected PROs have not done it in a way that facilitates their use in PCOR.

The launching of the Obamacare in the USA mandated the establishment of PCOR institute (PCORI). PCORI is committed specifically to the development and funding of comparative effectiveness researches (CERs), which measure patient-centered outcomes of any intervention. ${ }^{[10]}$ Large amount of the US budget is invested in the working of the PCORI. ${ }^{[1]}$ There are proposals to establish PCOR laboratories with aims to popularize PCOR in the US. ${ }^{[12]}$ PCORI is aiming to create a database of PCOR and PRO from CERs done in the past, and also to conduct fresh CERs that focus on PROs. This database is called the PCOR network.

\section{SUMMARY}

The shift from predominantly "disease-centered and doctorreported" model to a balanced model incorporating "patientcentered and patient-reported" outcomes is bound to happen, and PCOR is going to play a very important role in the transition. Increased awareness on the parts of clinicians, support of policy makers, and active participation of the informed patients are also crucial for this development. ${ }^{[1]}$

\section{REFERENCES}

1. Bayliss EA, Ellis JL, Shoup JA, Zeng C, McQuillan DB, Steiner JF. Association of patient-centered outcomes with patient-reported and ICD9-based morbidity measures. Ann Fam Med 2012;10:126-33.

2. Stanton MW. Expanding Patient-centered Care to Empower Patients and Assist Providers. Available from: http://www.ahrq.gov/research/findings/ factsheets/patient-centered/ria-issue5/index.html. [Last accessed on 2014 Sep 25].

3. Deshpande PR, Rajan S, Sudeepthi BL, Abdul Nazir CP. Patientreported outcomes: A new era in clinical research. Perspect Clin Res 2011;2:137-44.

4. Evans C. Closing the credibility gap in patient-reported outcomes. Expert Rev Pharmacoecon Outcomes Res 2002;2:1-2.

5. DeMuro C, Clark M, Mordin M, Fehnel S, Copley-Merriman C, Gnanasakthy A. Reasons for rejection of patient-reported outcome label claims: A compilation based on a review of patient-reported outcome use among new molecular entities and biologic license applications, 20062010. Value Health 2012;15:443-8.

6. Hostetter M, Klein S. Using Patient-reported Outcomes to Improve Healthcare Quality. Available from: http://www.commonwealthfund.org/ publications/newsletters/quality-matters/2011/december-january-2012/ in-focus. [Last accessed on 2014 Dec 19].

7. Gnanasakthy A, Mordin M, Clark M, DeMuro C, Fehnel S, CopleyMerriman C. A review of patient-reported outcome labels in the United States: 2006 to 2010. Value Health 2012;15:437-42.

8. Willke RJ, Burke LB, Erickson P. Measuring treatment impact: A review of patient-reported outcomes and other efficacy endpoints in approved product labels. Control Clin Trials 2004;25:535-52.

9. USFDA. Guidance for Industry. Patient-reported Outcome Measures: Use in Medical Product Development to Support Labelling Claims. Available from: http://www.fda.gov/downloads/Drugs/Guidances/UCM193282.pdf. [Last accessed on 2014 Sep 25].

10. Meisel ZF, Carr BG, Conway PH. From comparative effectiveness research to patient-centered outcomes research: Integrating emergency care goals, methods, and priorities. Ann Emerg Med 2012;60:309-16.

11. Yach D, Calitz C. New opportunities in the changing landscape of prevention. JAMA 2014;312:791-2.

12. James P, ADFM Research Development Committee, Pace W. Concept of a national primary care patient-centered outcomes research laboratory. Ann Fam Med 2013;11:282-3.

How to cite this article: Dang A, Vallish BN. The emerging role of patient-centered outcomes research in shaping the future of healthcare delivery in India: A brief review. Int J Med Public Health 2015;5:125-7.

Source of Support: Nil, Conflict of Interest: None declared. 